\title{
Electron acceleration in laboratory-produced turbulent collisionless shocks
}

F. Fiuza ${ }^{1 *}$, G. F. Swadling ${ }^{2}$, A. Grassi ${ }^{1}$, H. G. Rinderknecht ${ }^{3}$, D. P. Higginson ${ }^{2}$, D. D. Ryutov ${ }^{2}$, C. Bruulsema $^{4,1}$, R. P. Drake ${ }^{5}$, S. Funk ${ }^{6}$, S. Glenzer ${ }^{1}$, G. Gregori ${ }^{7}$, C. K. Li ${ }^{8}$, B. B. Pollock ${ }^{2}$, B. A. Remington $^{2}$, J. S. Ross ${ }^{2}$, W. Rozmus ${ }^{1,4}$, Y. Sakawa ${ }^{9}$, A. Spitkovsky ${ }^{10}$, S. Wilks ${ }^{2}$, H.-S. Park ${ }^{2}$

${ }^{1}$ High Energy Density Science Division, SLAC National Accelerator Laboratory, Menlo Park, CA 94025, USA

${ }^{2}$ Lawrence Livermore National Laboratory, Livermore, CA 94551, USA

${ }^{3}$ Laboratory for Laser Energetics, University of Rochester, 250 E. River Road, Rochester, NY 14623, USA

${ }^{4}$ Department of Physics, University of Alberta, Edmonton, Alberta T6G 2E1, Canada

${ }^{5}$ University of Michigan, Ann Arbor, MI 48109, USA

${ }^{6}$ Friedrich-Alexander-Universitat Erlangen-Nuremberg, Erlangen Centre for Astroparticle Physics, 91058 Erlangen, Germany

${ }^{7}$ Department of Physics, Oxford University, Parks Road, OX1 3PU, United Kingdom

${ }^{8}$ Massachusetts Institute of Technology, Cambridge, MA 02139, USA

${ }^{9}$ Osaka University, Osaka 565-0871, Japan

${ }^{10}$ Princeton University, Princeton, NJ 08544, USA

*fiuza@slac.stanford.edu 
Astrophysical collisionless shocks are among the most powerful particle accelerators in the Universe. Generated by violent interactions of supersonic plasma flows with the interstellar medium, supernova remnant shocks are observed to amplify magnetic fields ${ }^{1}$ and accelerate electrons and protons to highly relativistic speeds ${ }^{2-4}$. In the well-established model of diffusive shock acceleration ${ }^{5}$ relativistic particles are accelerated by repeated shock crossings. However, this requires a separate mechanism that pre-accelerates particles to enable shock crossing. This is known as the 'injection problem', which is particularly relevant for electrons, and remains one of the most important puzzles in shock acceleration ${ }^{6}$. In most astrophysical shocks, the details of the shock structure cannot be directly resolved, making it challenging to identify the injection mechanism. Here, we report results from laser-driven plasma flow experiments, and related simulations, that probe the formation of turbulent collisionless shocks in conditions relevant to young supernova remnants. We show that electrons can be effectively accelerated in a first-order Fermi process by small-scale turbulence produced within the shock transition to relativistic non-thermal energies, helping overcome the injection problem. Our observations provide new insight on electron injection at shocks and open the way for controlled laboratory studies of the physics underlying cosmic accelerators.

Most astrophysical shocks are collisionless, meaning that they are formed by plasma instabilities that dissipate flow energy via magnetic field amplification, plasma heating, and particle acceleration $^{6,7}$. Particle injection is thus closely related to the shock formation mechanism and the properties of the turbulent magnetic field produced at the shock. These processes are generally controlled by the shock Mach number (the ratio of the shock velocity to the ambient Alfvén or 
sound speed) in ways which are not yet well understood. In situ spacecraft measurements of the Earth's bow shock have long shaped our understanding of collisionless shocks at moderate Alfvén Mach numbers, $M_{A} \sim 3-10^{8}$. However, our knowledge of the very high Mach number regime $\left(M_{A} \gg 10\right)$ relevant to supernova remnant (SNR) shocks is significantly more limited, given the poorly constrained local conditions at these exotic, distant shocks, and has mostly been gained from numerical simulations ${ }^{9-12}$.

In the last decade, there has been a significant effort in using kJ-class lasers to produce supersonic, superalfvenic plasma flows in the laboratory ${ }^{13-20}$ that would enable controlled studies of collisionless shocks in conditions that can be directly scaled to astrophysical environments ${ }^{21}$ and help validate numerical and theoretical models. Several works have focused on pre-magnetized or piston-driven plasmas, where the ambient magnetic field compresses the incoming plasma flows but no turbulence is present ${ }^{18,19}$, or on low-Mach number shocks where particle acceleration is less efficient ${ }^{20}$. The study of high-Mach number turbulent shocks in conditions relevant to young ( $<1000$ year-old) SNRs, where the ambient magnetic field is not dynamically important and the shock is mediated by electromagnetic instabilities has so far remained elusive given the need to drive the plasma interaction for significantly longer times ${ }^{22}$. Important progress towards this goal was made by experiments at the OMEGA laser facility that demonstrated the development of strong magnetic fields by Weibel-type filamentation instabilities ${ }^{15,16}$; yet, for the available $1-10 \mathrm{~kJ}$ laser energy, the formation of collisionless shocks could not be observed. Here, we report on laboratory astrophysics experiments at unprecedented megajoule (MJ) laser energy that probe the formation of high- $M_{A}$ turbulent collisionless shocks and the acceleration of electrons to relativistic energies 
that exceed by a factor of more than 100 the electron thermal energy at the shock.

The experiments were conducted at the National Ignition Facility. Symmetric and counterstreaming plasma flows are produced by laser-ablation of two deuterated carbon $\left(\mathrm{CD}_{2}\right)$ targets separated by $L_{\text {system }}=2.5 \mathrm{~cm}$. Each target is irradiated by 84 laser beams, delivering $0.455 \mathrm{MJ}$ of $351 \mathrm{~nm}$ light to a surface area of $0.5 \mathrm{~cm}^{2}$. The interaction of the plasma flows is characterized using a suite of diagnostics that includes optical Thomson scattering (OTS) of a probe beam, X-ray selfemission from the plasma, and electron spectrometers (see Figure 1 and Methods). In Figure 2, we present OTS measurements of the evolution of electron density and temperature of the plasma in the mid-plane region between the targets obtained in the time interval $6-30 \mathrm{~ns}$ from the beginning of laser irradiation. The properties of a single, independent flow are characterized by irradiating only one target. Its electron density varies between $n_{e} \sim 2-5 \times 10^{19} \mathrm{~cm}^{-3}$ and its electron temperature is $k_{B} T_{e} \sim 0.5 \mathrm{keV}$ over the span of the $24 \mathrm{~ns}$ measurement. When the two flows are allowed to interact (by irradiating both targets), their plasma properties change profoundly. Between 6 and 12 ns a strongly compressed zone with plasma density $\gtrsim 4 \times$ the single flow density is formed near the mid-plane. During the same time, the plasma electrons are heated in this region to $3 \mathrm{keV}$. Measurements of the X-ray self-emission from the plasma confirm that when the two flows interact a strongly compressed and heated zone is formed, consistent with the OTS data, with a transverse radius of $\sim 0.5 \mathrm{~cm}$ (see Figure 1c, Extended Data Figure ??, and Supplementary Table 1).

The measured density compression demonstrates the formation of high-Mach number col- 
lisionless shocks, for which the hydrodynamic jump conditions predict a compression factor ${ }^{23}$ $n_{2} / n_{1} \simeq 4$, consistent with our observations $\left(n_{2}\right.$ and $n_{1}$ are the downstream and upstream densities). Note that if the two flows would freely interpenetrate, the resulting density would simply double. The velocity of the laser-produced plasma flows at the mid-plane distance is given by the self-similar expansion theory ${ }^{24}, v_{\text {flow }}=c_{S 1}+L_{\text {system }} /(2 t) \simeq 200+12500 / t[\mathrm{~ns}] \mathrm{km} / \mathrm{s}$, as verified in previous experiments ${ }^{14}$, with $t$ being the time from the laser irradiation and $c_{S 1}$ the upstream sound speed. At $t \sim 8 \mathrm{~ns}$, when the shock compression is observed, the unperturbed flow velocity is $v_{\text {flow }} \sim 1800 \mathrm{~km} / \mathrm{s}$, corresponding to a ion-ion collisional mean free path ${ }^{25}$ of $\sim 80 \mathrm{~cm}$, which is much larger than our system, clearly indicating that the shock is collisionless (see Supplementary Information for detailed discussion on collisionality). The magnetic field carried by the laser-produced plasma flows is very weak $(\approx 20 \mathrm{kG})$ and from the measured plasma parameters we infer that the shock sonic and Alfvén Mach numbers are $M_{S} \sim 12$ and $M_{A} \sim 400$, respectively (see Supplementary Information). Our experiments thus probe shock conditions relevant to young SNRs (e.g., Tycho, SN1006, and Cas A), with typical shock velocities of a few 1000 $\mathrm{km} / \mathrm{s}$ and $M_{A} \gg 10$, as illustrated in Table 1 , for which shock formation must be mediated by electromagnetic fields produced during the interpenetration of the plasma flows.

The formation of collisionless shocks in the weakly magnetized regime of our experiments is mediated by the Weibel instability ${ }^{26,27}$. This is confirmed by large-scale two-(2D) and threedimensional (3D) particle-in-cell (PIC) simulations of the interaction of the flows for our experimental conditions (see Methods), as illustrated in Figure 3. The ion Weibel instability grows due to the velocity anisotropy of the counter-streaming flows, producing flow-aligned filamentary 
currents in the plasma threaded by magnetic fields. At the beginning of the interaction $(t \sim 6$ $\left.\mathrm{ns}, v_{\text {flow }} \sim 2200 \mathrm{~km} / \mathrm{s}\right)$, the instability growth time is $\tau_{W} \simeq c /\left(v_{\text {flow }} \omega_{p i}\right) \sim 0.02 \mathrm{~ns}$, where $\omega_{p i}=\left(4 \pi n_{i} Z^{2} e^{2} / m_{i}\right)^{1 / 2}$ is the ion plasma frequency, $Z e$ is the ion charge, $m_{i}$ is the ion mass, and $c$ is the speed of light. Within just $0.2 \mathrm{~ns}$, this instability produces filamentary magnetic fields that reach $1 \mathrm{MG}$, with a transverse wavelength of $50 \mu \mathrm{m}\left(\sim\right.$ ion skin depth $c / \omega_{p i}$, as expected from linear theory). Such strong fields correspond to $\sim 1 \%$ of the flow kinetic energy (Fig. 3c). This is consistent with the basic scaling laws ${ }^{21}$ for the magnetic field amplification observed in previous experiments under similar geometry but lower laser energy drive ${ }^{15,16}$, which indicate that MG-level magnetic fields must be produced in our experiments. After the saturation of the Weibel instability, the current filaments can be subject to secondary instabilities, merging towards larger scales $^{28}$ and breaking ${ }^{29}$, leading to the onset of magnetic turbulence. Our simulations indicate that the characteristic spatial scale of the turbulent magnetic field grows to $L_{B} \sim 200 \mu \mathrm{m}$ (Fig. $3 \mathrm{~b}$ ), which is comparable to the ion gyroradius $r_{g i} \sim 300 \mu \mathrm{m}$. The flows can then be efficiently compressed within a few ion gyroperiods $\left(\tau_{g i}=0.2 \mathrm{~ns}\right)$. Fully formed shocks are observed at $9 \mathrm{~ns}$, consistent with experimental measurements (Fig. 3d).

Shock formation is accompanied by the observation of very energetic non-thermal electrons. Electron spectrometer measurements indicate that when the two flows collide electrons are accelerated up to $500 \mathrm{keV}$ (Fig. 4a), which exceeds by more than a factor 100 the thermal energy of the shocked plasma $\left(k_{B} T_{e} \sim 3 \mathrm{keV}\right)$. When only one of the flows is produced, the measured electron spectrum is vastly different, showing a significantly lower number $(<8 \%)$ and energy $(<3 \%)$ of electrons above $30 \mathrm{keV}$, when compared to the double flow case. These lower energy electrons 
observed in a single flow are produced by the laser-plasma interaction, and their number should simply double in the case of two plasma flows (see Supplementary Information). Therefore, our measurements indicate that a different acceleration mechanism is present for the interpenetrating plasmas and that the bulk plasma distribution, which dominates at energies below those observed in the spectrometer, should act as a seed for the large number of high-energy electrons measured.

The simulations of the experimental interaction confirm the ability of the shocks to accelerate electrons from the thermal pool to $>100 k_{B} T_{e}$ on the time scale of the experiment (Fig. $\left.4 \mathrm{~b}\right)$. The non-thermal electron distribution in the shocked plasma region evolves towards a power-law energy tail $\epsilon^{-p}$ with spectral index $p \sim 3$. However, we note that the detailed shape of the non-thermal spectra obtained in simulations (Fig. 4b) cannot be straightforwardly compared with experiments (Fig. 4a) given that the latter measure the time-integrated spectrum of only the escaping electrons, which is modified by the energy-dependent escape time associated with electron diffusion (see Supplementary Information). In order to further elucidate the electron acceleration process, a random sample of 200 electrons is chosen from the non-thermal tail obtained in the simulation at $13 \mathrm{~ns}$ and their acceleration history is analyzed in detail. We observe that electrons are rapidly accelerated - on a ns time scale - as they are trapped within one shock transition layer and suffer multiple reflections by the turbulent fields produced by the Weibel instability (Fig. 4c). The average fractional energy gains experienced upon each reflection are $\Delta \epsilon / \epsilon \sim v_{\text {flow }} / v$, with $v$ the electron velocity, consistent with a first-order Fermi process (see Fig. 4d and Methods).

The measurement of non-thermal electrons with up to $500 \mathrm{keV}$ from colliding plasma flows 
is indeed consistent with electron acceleration by magnetic turbulence produced at the shock transition layer. The gyroradius of $500 \mathrm{keV}$ electrons in $\mathrm{MG}$ magnetic fields is $\sim 30 \mu \mathrm{m}$, which is significantly smaller than the shock transition $L_{s h} \gtrsim r_{g i} \sim 300 \mu \mathrm{m}$. Thus, the observed high-energy electrons could not have accessed standard DSA via multiple shock crossings. For electrons trapped in the shock transition region, the maximum electron energy is limited by the transverse size of the shock, $R_{s h} \sim 0.5 \mathrm{~cm}$, and corresponds to the largest potential difference a particle can experience in the shock before escaping transversely (known as the Hillas limit ${ }^{30}$ ) $\epsilon_{\max }=e E R_{s h} \sim e B R_{s h} v_{\text {flow }} / c$, where $E \sim B v_{\text {flow }} / c$ is the electric field associated with the turbulent magnetic fields advected by the flows into the shock. For the conditions of our experiments ( $B \sim 1 \mathrm{MG}, v_{\text {flow }} \sim 1000 \mathrm{~km} / \mathrm{s}$ ), we obtain $\epsilon_{\max } \sim 500 \mathrm{keV}$, consistent with the experimental measurements.

Our results provide compelling evidence that magnetic turbulence produced in high-Mach number shocks can accelerate electrons to relativistic non-thermal energies, and thus help overcome the injection problem. These findings are relevant to high-Mach number astrophysical shocks, such as those found in young SNRs, and will stimulate the development of better injection models for those systems. In the future, the developed experimental platform can be adapted to different shock configurations, including those with an ambient external magnetic field of variable strength and orientation. This opens a path for controlled studies of shocks in regimes that can greatly complement both astrophysical observations and spacecraft measurements and help validate particle acceleration models. 


\section{Methods}

Experimental setup Experiments were conducted on the National Ignition Facility (NIF) at the Lawrence Livermore National Laboratory. Double flow experiments used a pair of counter-facing targets (consisting of $1 \mathrm{~mm}$ thick, $8 \mathrm{~mm}$ diameter $\mathrm{CD}_{2}$ disks) separated by $25 \mathrm{~mm}$. The central facing surface of each disk was heated by 84 laser beams with $3 \mathrm{~ns}$ square top duration and $351 \mathrm{~nm}$ wavelength, delivering a total of $0.455 \mathrm{MJ}$ per target. Standard NIF phase plates were used, and the beams were tiled and defocused over the surface of the target disks to produce an approximately flat-topped intensity distribution over the $\sim 7 \mathrm{~mm}$ diameter central region of the surface, resulting in an average intensity $\sim 4 \times 10^{14} \mathrm{Wcm}^{-2}$. The surfaces of the heated targets expand rapidly producing high velocity ablation-plasma outflows. In double flow experiments the two targets are irradiated simultaneously to produce two symmetric counter-streaming plasma flows that interact near their midplane. In single flow experiments, only one target is used and irradiated with the same laser conditions. The plasma properties are characterized near the midplane between the two targets using a suite of diagnostics. The main diagnostics are temporally resolved optical Thomson scattering (OTS) of a $351 \mathrm{~nm}$ probe beam, time-gated X-ray self-emission from the plasma (GXD), and an electron spectrometer (NEPPS), which are positioned along the midplane as indicated in the Supplementary Figure 1.

Thomson scattering measurements The parameters of the plasmas produced in the experiments were characterized using the NIF optical Thomson scattering diagnostic, at a point $12.5 \mathrm{~mm}$ from the surface of the target, both in the case of a single flow and double flow. In the double flow 
experiments this position corresponds to the center of the experiment. Two $351 \mathrm{~nm} \mathrm{f} / 20.6 \mathrm{NIF}$ beams were used to supply the Thomson scattering probe. The beams enter the system from (polar, azimuthal) angle $(137.8,13.2)$ and scattered light is collected along $(90,315)$ giving a scattering angle of $\sim 71^{\circ}$. The Thomson probe beams (24ns, $8 \mathrm{~kJ}$ per beam) used $400 \mu \mathrm{m}$ phase plates to reduce the size of the scattering volume. Beam spots were tiled side by side to reduce the required pointing tolerance for the OTS diagnostic, so that the probe intensity was $6.6 \times 10^{13}$ $\mathrm{Wcm}^{-2}$. The diameter of the spectrometer collection cone at focus is $50 \mu \mathrm{m}$, defined by a pinhole aperture at the entrance to the spectrometers. The OTS volume therefore takes the form of a cylinder, with a dimension of $50 \mu \mathrm{m}$ in the direction of the flows and $50 \mu \mathrm{m}$ and $400 \mu \mathrm{m}$ in the transverse directions. The resulting probed k-vector, shown in green in Supplementary Figure 1, lies at an angle $49.3^{\circ}$ from the vector of approach between the two ablation flows. Scattered light was collected at f/12 using an off-axis Schwarzschild objective and fed into an optical grating spectrometer $\left(G=1200 \mathrm{~mm}^{-1}, \mathrm{f}=0.15 \mathrm{~m}, \delta \lambda=1 \mathrm{~nm}\right)$. The spectrum is recorded using a Ross optical streak camera, with a sweep window of $30 \mathrm{~ns}$ and a temporal resolution $\delta t \sim 30 \mathrm{ps.} \mathrm{The}$ spectrometer has a bandwidth of $\sim 65 \mathrm{~nm}$; central wavelength was $300 \mathrm{~nm}$ for the single flow measurement and $290 \mathrm{~nm}$ for the double flow experiment. The OTS probe beam had a pulse duration of $24 \mathrm{~ns}$, and was turned on at $6 \mathrm{~ns}$.

We observe the electron plasma wave (EPW or Langmuir) feature of the Thomson scattering spectrum, for which the electron temperature $\left(T_{e}\right)$ and density $\left(n_{e}\right)$ affect the spectrum in different ways. The wavelength shift for the peak of the EPW feature is dominated by the electron density and only weakly sensitive to $T_{e}$. On the other hand, an increase in temperature broadens 
the peak due to Landau damping. This can significantly constrain the combination of density and temperature capable of matching the measured spectrum and allows for these properties to both be determined simultaneously ${ }^{31}$. The plasma parameters were inferred by fitting the measured OTS spectrum with the theoretical OTS scattering cross section $S(k, \omega)=(2 \pi / k) f_{M}(\omega / k) /|\epsilon(k, \omega)|^{2}$, where $\epsilon(k, \omega)$ is the longitudinal plasma dielectric function evaluated at the probed $\mathrm{k}$-vector $k$ and frequency $\omega$ of the electron density fluctuations, $f_{M}(\omega / k)$ is the $1 \mathrm{D}$ electron Maxwellian distribution function projected on the direction of $k$ and evaluated at the resonant velocity corresponding to the phase velocity of fluctuations. This calculated cross-section was corrected using a spectrometer response calibration curve which was measured prior to the shot. The plasma was modeled as a deuteron / carbon plasma with a single Maxwellian electron distribution. The amplitude, dc background, $T_{e}$ and $n_{e}$ were varied to achieve the best fit possible. To determine the errors in $n_{e}$ and $T_{e}$, we used a Newton-Gauss fit with parameter errors determined by the shape of the $\chi^{2}$ value as a function of the fit parameters. This includes the covariance of the system in the parameter errors. The error in density and temperature obtained from this analysis ranges between $3-10 \%$. Supplementary Figure 2 illustrates the results of this procedure, including examples of the best fits to our data and how they change for different combinations of $n_{e}$ and $T_{e}$. The error bars indicated in the measurements of Figure 2 take into account the errors in the fitting of the OTS spectrum, an absolute error in the central wavelength (offset error) of $1 \mathrm{~nm}$, and a magnification error of $2 \%$ introduced by the streak camera electron optics.

Electron spectrometer measurements The NIF Electron Positron Proton Spectrometer (NEPPS) records electron spectra in the range $30-900 \mathrm{keV}$ by deflecting electrons incident through a $1 \mathrm{~mm}$ 
diameter aperture by a permanent magnetic field onto a BAS-SR image plate. The diagnostic is identical to that described in Ref. ${ }^{32}$ with the exception of a reduced peak magnetic field strength at 280 Gauss. The dispersion relation was simulated by using finite element (COMSOL) analysis to calculate the 3D magnetic field and electron trajectories in the detector, and was calibrated using benchtop measurements of the peak field strength. The NEPPS spectrometer was fielded along a line of sight perpendicular to the axis of flow, with the aperture positioned $55 \mathrm{~cm}$ from the target axis. Data were analyzed by integrating the signal perpendicular to the dispersion direction over the data region, and accounting for calibrated image plate response and solid angle to evaluate the number of electrons per keV per steradian. We note that the accelerated electrons in the shocked region are expected to have an isotropic distribution and this has also been confirmed in our simulations of the experiment. However, the escape of electrons is expected to be energy dependent, as discussed below, and could be anisotropic. A spatially-dependent background was evaluated by averaging in the non-signal region and was subtracted from the data. The peak signal to background ratio in the signal region was $\sim 6$ in the double-flow experiments. The two double flow spectra shown in Fig. 4a illustrate the typical results obtained. In single flow experiments the signal measured was close to the background level. In Fig. 4a we show the single flow spectrum with the largest signal among single flow experiments. The order-of-magnitude increase in both the number and energy of electrons observed in the double flow spectra as compared to the single flow spectra was reproducible in all experiments. The largest uncertainty in the analysis is a systematic error in the image plate response, which is approximately $25 \%$ across the energy range used ${ }^{33}$, as indicated by the shaded region in Fig. 4a. 
X-ray detector measurements A Gated X-ray Detector (GXD) images the self-emitting X-rays from the interacting plasma flows using an array of pinholes and differential filters. It has four strips of time resolving areas such that the timing on each strip can be independently controlled. The magnification is set to $2.02 \times$ and the pinhole diameter is $15 \mu \mathrm{m}$. Two filters are used in each strip $-0.5 \mu \mathrm{m}$ Nickel (1/3 of strip area) and $2 \mu \mathrm{m}$ Vanadium (2/3 of strip area) backed by $8 \mu \mathrm{m}$ acrylic. This setting is sensitive to $5-7 \mathrm{keV}$ emission. The timing for each strip was set to 5, 12, 15, and $20 \mathrm{~ns}$, respectively. Each strip spanned $\sim 600 \mathrm{ps}$ integration time. The same exact filtering and timing were used for both single and double flow experiments to compare their relative brightness. Extended Data Figure ?? shows examples of lineouts (averaged over a $900 \mu \mathrm{m}$ width) of X-ray emission along the mid-plane region of the experiment at $15 \mathrm{~ns}$. The measured ratio of Xray signal between double and single flow experiments has been compared with the ratio expected from the Thomson scattering measurements. We consider the bulk plasma in the measurement region (central region of the interaction) to have a Maxwell-Boltzmann distribution, which results in a bremsstrahlung emission power per unit energy and volume that scales as $T_{e}^{-1 / 2} n_{e}^{2} e^{-\epsilon_{\gamma} /\left(k_{B} T_{e}\right)}$ ${ }^{34}$, with $\epsilon_{\gamma}$ the photon energy. The Thomson scattering measurements provide local, time-resolved electron density and temperature information in a small volume at the center of the region viewed by the GXD. Using these measured values, the bremsstrahlung spectrum can be calculated for each $\mathrm{X}$-ray measurement time in each experiment. The framing camera response is calibrated to $20 \mathrm{keV}$ and transmission data for the Kapton, $\mathrm{Ni}$, and $\mathrm{V}$ filters in front of the GXD are available through CXRO. Convolving the calculated bremsstrahlung emission, the filter transmission, and the GXD response curve, relative signal levels through each filter configuration have been estimated for each 
experiment. Supplementary Table 1 shows the comparison of the X-ray signal ratio predicted from OTS data and the ratios measured from GXS for both V and Ni filter channels at 15 and $20 \mathrm{~ns}$. In both cases, analysis of the OTS data indicates that the double flow X-ray signal should be $\sim 100$ times brighter than the single flow and this is consistent with the measured X-ray ratios. The X-ray signal ratios cannot be confidently measured at 5 and 12 ns because at 5 ns the lasers had just turned off and the strip is saturated and at $12 \mathrm{~ns}$ there is significant signal bleed from the $5 \mathrm{~ns}$ strip of the framing camera, which affects primarily the single flow measurement.

Radiation-hydrodynamic simulations The radiation-hydrodynamics code HYDRA ${ }^{35}$ is used to model the laser absorption and plasma ablation of a single foil. The laser irradiation follows the experimental setup by including individual lasers with accurate beam pointing, incident angles, pulse durations, and focal spot sizes to obtain the expected on-target intensity. A multiplier of 0.6 on the total laser energy (corresponding to the laser absorption efficiency) is used, based on the benchmarks of HYDRA simulations with previous experimental data on OMEGA. The obtained velocity and density profiles are shown in Supplementary Figure 3. The velocity of the expansion is found to fit well with self-similar theory ${ }^{24}$ with peak velocities above $2000 \mathrm{~km} / \mathrm{s}$. The obtained density profile is in good agreement (within 20\%) with the experimental Thomson scattering measurements.

Particle-in-Cell simulations Two-dimensional (2D) and three-dimensional (3D) particle-in-cell (PIC) simulations of the plasma flow interaction are performed with the fully electromagnetic, fully relativistic, and massively parallel PIC code OSIRIS 4.0 $0^{36,37}$. The PIC simulations are initialized 
using the laser-ablated plasma profiles obtained from HYDRA before the interaction of the flows. The interaction starts at $6 \mathrm{~ns}$ from the laser irradiation and it is described fully kinetically. Given the computational expense of kinetic simulations for the large temporal and spatial scales of the experiments, the majority of the simulations performed were $2 \mathrm{D} 3 \mathrm{~V}$ and used a reduced ion to electron mass ratio in the range $m_{i} /\left(m_{e} Z\right)=32-512$. A 3D3V simulation with $m_{i} /\left(m_{e} Z\right)=32$ was performed to test the overall impact of 3D effects. The initial flow velocity was scaled up from the experiment by a factor 30 (i.e., an experimental flow velocity of $1000 \mathrm{~km} / \mathrm{s}$ corresponds to $0.1 c$ in the simulation). This is based on the fact that for the conditions of our study the physics of the electromagnetic instabilities that mediate the shock formation and particle acceleration is independent of the flow velocity, provided it is non-relativistic. The evolution of two systems, $S_{1}$ and $S_{2}$, dominated by electromagnetic processes and with flow velocities $v_{1}$ and $v_{2}$ is the same at times $t_{2}=t_{1} v_{1} / v_{2}{ }^{21}$. This scaling is used to convert the simulation time into the experimental time. The reference simulation uses $m_{i} /\left(m_{e} Z\right)=128$ (Figures 3 and 4). The simulation box size is $L_{x}=972 c / \omega_{p i 0} \simeq 3.1 \mathrm{~cm}$ by $L_{y}=71 c / \omega_{p i 0} \simeq 0.23 \mathrm{~cm}$, where $\omega_{p i 0}$ is the ion plasma frequency corresponding the reference plasma density $n_{0}=10^{20} \mathrm{~cm}^{-3}$ (approximately the density of the shocked plasma). This domain is resolved by $31500 \times 2300$ cells, corresponding to a resolution of $0.35 c / \omega_{p e 0}$. Each plasma flow is modeled using 50 macroparticles per cell with cubic particle shapes for improved numerical accuracy. The 3D simulation used the same resolution and particle shape with 4 macroparticles per cell. The boundary conditions for both particles and fields are open along the flow direction and periodic transversely. We have tested the numerical results in $2 \mathrm{D}$ by varying the resolution $\left(0.07-0.35 c / \omega_{p e 0}\right)$, the number of particles per cell $(18-$ 
$200)$, and the ion to electron mass ratio $(32-512)$, observing overall agreement on the shock structure. We have also checked that the dominant electron acceleration mechanism discussed in this work does not depend significantly on the ion to electron mass ratio, for the large ratios used in our simulations. In particular, we observe that when normalized to the thermal energy of the shocked electrons, the non-thermal electron spectrum is identical for the range of mass ratios tested (Supplementary Figure 4). Based on this observation, the simulated electron energy in Figures $4 \mathrm{c}, \mathrm{d}$ is converted into experimental units according to the measured electron temperature at the shock $k_{B} T_{e}=3 \mathrm{keV}$. The simulation used to illustrate the experimental setup in Figure 1a has a larger transverse box size of $3.1 \mathrm{~cm}$, a reduced mass ratio $m_{i} /\left(m_{e} Z\right)=32$, and open boundaries transversely. This simulation captures the finite transverse size of the plasma flows and shock structure. Additional PIC simulations using the measured single flow plasma profile have been performed and compared with those using the HYDRA predicted plasma profile (maximum $20 \%$ variation), showing a similar shock structure. Finally, a 1D PIC simulation using a Monte-Carlo Coulomb collision operator was performed for the same plasma profile of the reference simulation. This simulation does not capture the Weibel instability (which is a transverse instability) and can thus isolate the role of Coulomb collisions in the slow down and compression of the flows. It includes collisions between all plasma species (electrons and ions) and indicates that collisional effects only start impacting the flow interpenetration after $22 \mathrm{~ns}$, and thus after the time scale of the shock physics studied in this work.

Analysis of non-thermal electron acceleration from the simulations In order to understand how the non-thermal electrons observed in the simulations are accelerated, we track the detailed 
trajectories and energy gains of a representative sample. We randomly choose 200 electrons from the non-thermal tail $\left(\epsilon>30 k_{B} T_{e}\right)$ at $13 \mathrm{~ns}$ (shown in Fig. $\left.4 \mathrm{~b}\right)$ and then repeat the exact same simulation tracking the evolution of these electrons from thermal to non-thermal. We observe that electrons are accelerated primarily as they are trapped in the transition layer of one shock and experience large energy gains from reflections along the flow axis, as illustrated in Fig. 4c. We calculate the energy variation from reflections in the x-direction (identified by the change in the sign of the electron velocity $v_{x}$ ), including both energy gaining and energy losing reflections, for electrons with energies above the thermal energy $\left(\epsilon>k_{B} T_{e}\right)$. By probing the electron trajectory with a period $\gtrsim \tau_{g e}$ (with $\tau_{g e}$ the electron gyroperiod) we can properly capture the individual reflections and we have verified that the results do not change significantly by changing this period in the range $1-5 \tau_{g e}$. We bin the electrons by energy and calculate the average energy change $\Delta \epsilon$ for bins with more than 10 reflections. We find that the majority of reflections are energy gaining and that the average fractional energy change per reflection is $\Delta \epsilon / \epsilon \sim v_{\text {flow }} / v$ (Fig. 4d), with $v_{\text {flow }}$ the average flow velocity in the shock transition region. This indicates that electrons are accelerated via a first-order Fermi process.

Comparison between laboratory shock parameters and young SNRs Despite the enormous difference in physical scale and density between laboratory and astrophysical systems, the similarity of the dimensionless plasma parameters indicates that the dominant plasma processes can be directly scaled between both systems ${ }^{21}$. A comparison between the parameters of our laboratory experiments and those of typical young SNRs (e.g. Tycho, SN 1006, and Cas A) are presented in Table 1. The shock sonic and Alfvén Mach numbers are calculated as $M_{S}=v_{\mathrm{sh}} / c_{S 1}$ and 
$M_{A}=v_{\mathrm{sh}} / v_{A}$, where $v_{\mathrm{sh}}=v_{\text {flow }} /\left(1-n_{1} / n_{2}\right)$ is the shock velocity measured in the frame of the upstream flow, $c_{S 1}=\left(\gamma Z k_{B} T_{e} / m_{i}\right)^{1 / 2}$ is the upstream sound speed, and $v_{A}=B /\left(4 \pi n_{i} m_{i}\right)^{1 / 2}$ is the upstream Alfvén speed, with $\gamma=5 / 3$ the adiabatic index, $n_{i}$ the ion density of the flow, and $Z$ the ion charge number.

Data Availability The data represented in Fig. 2, Fig. 3c-d, and Fig. 4 are provided with the paper as source data. All other data that support the plots within this paper and other findings of this study are available from the corresponding author upon reasonable request.

Code Availability The PIC code OSIRIS ${ }^{36,37}$ used in this study can be obtained from the OSIRIS Consortium, consisting of UCLA and IST (Portugal).

Acknowledgments The authors thank M. Hohenberger and G. Fiksel for their assistance in the analysis of FFLEX and NEPPS data, respectively. This work was supported by the U.S. Department of Energy SLAC Contract No. DE-AC02-76SF00515 and Lawrence Livermore National Laboratory Contract No. DE-AC52-07NA27344, the U.S. DOE Early Career Research Program under FWP 100331, the U.S. DOE Office of Science, Fusion Energy Sciences under FWP 100182, the LLNL Laboratory Directed Research and Development Program grant 15-ERD065, and the Engineering and Physical Sciences Research Council of the United Kingdom (grant numbers EP/M022331/1 and EP/N014472/1). The authors also acknowledge the OSIRIS Consortium, consisting of UCLA and IST (Portugal) for the use of the OSIRIS 4.0 framework and the visXD framework. Simulations were run on Mira and Theta (ALCF) through ALCC awards and on Vulcan and Quartz (LLNL) through grand challenge awards. 
Author Contributions F.F. and H.S.P. conceived and led this project. The experiments were designed and carried out by G.S., H.R., H.S.P., and F.F. The data was analyzed by G.S., H.R., C.B., B.P. and F.F. Numerical simulations were performed by A.G., F.F., D.H., and S.W. Additional theoretical support was provided by D.D.R., W.R., A.S., and G.G. The paper was written by F.F. with contributions from all the authors.

Competing interests The authors declare no competing interests.

1. Völk, H. J., Berezhko, E. G. \& Ksenofontov, L. T. Magnetic field amplification in tycho and other shell-type supernova remnants. A\&A 433, 229-240 (2005).

2. Koyama, K. et al. Evidence for shock acceleration of high-energy electrons in the supernova remnant SN1006. Nature 378, 255-258 (1995).

3. Aharonian, F. A. et al. High-energy particle acceleration in the shell of a supernova remnant. Nature 432, 75-77 (2004).

4. Ackermann, M. et al. Detection of the characteristic pion-decay signature in supernova remnants. Science 339, 807-811 (2013).

5. Blandford, R. \& Eichler, D. Particle acceleration at astrophysical shocks: A theory of cosmic ray origin. Physics Reports 154, 1-75 (1987).

6. Treumann, R. A. Fundamentals of collisionless shocks for astrophysical application, 1. nonrelativistic shocks. The Astronomy and Astrophysics Review 17, 409-535 (2009). 
7. Sagdeev, R. Z. Cooperative Phenomena and Shock Waves in Collisionless Plasmas. Reviews of Plasma Physics 4, 23 (1966).

8. Oka, M. et al. Electron scattering by high-frequency whistler waves at earth's bow shock. The Astrophysical Journal 842, L11 (2017).

9. Hoshino, M. \& Shimada, N. Nonthermal electrons at high mach number shocks: Electron shock surfing acceleration. The Astrophysical Journal 572, 880-887 (2002).

10. Spitkovsky, A. Particle acceleration in relativistic collisionless shocks: Fermi process at last? The Astrophysical Journal 682, L5-L8 (2008).

11. Kato, T. N. \& Takabe, H. Nonrelativistic Collisionless Shocks in Unmagnetized Electron-Ion Plasmas. Astrophysical Journal 681, L93-L96 (2008).

12. Matsumoto, Y., Amano, T., Kato, T. N. \& Hoshino, M. Stochastic electron acceleration during spontaneous turbulent reconnection in a strong shock wave. Science 347, 974-978 (2015).

13. Kugland, N. L. et al. Self-organized electromagnetic field structures in laser-produced counterstreaming plasmas. Nature Physics 8, 809-812 (2012).

14. Ross, J. S. et al. Characterizing counter-streaming interpenetrating plasmas relevant to astrophysical collisionless shocks. Physics of Plasmas 19, 056501 (2012).

15. Fox, W. et al. Filamentation instability of counterstreaming laser-driven plasmas. Phys. Rev. Lett. 111, 225002 (2013). 
16. Huntington, C. M. et al. Observation of magnetic field generation via the Weibel instability in interpenetrating plasma flows. Nature Physics 11, 173-176 (2015).

17. Ross, J. S. et al. Transition from collisional to collisionless regimes in interpenetrating plasma flows on the national ignition facility. Phys. Rev. Lett. 118, 185003 (2017).

18. Schaeffer, D. B. et al. Generation and evolution of high-mach-number laser-driven magnetized collisionless shocks in the laboratory. Phys. Rev. Lett. 119, 025001 (2017).

19. Li, C. K. et al. Collisionless shocks driven by supersonic plasma flows with self-generated magnetic fields. Phys. Rev. Lett. 123, 055002 (2019).

20. Rigby, A. et al. Electron acceleration by wave turbulence in a magnetized plasma. Nature Physics 14, 1745-2481 (2018).

21. Ryutov, D. D. et al. Basic scalings for collisionless-shock experiments in a plasma without pre-imposed magnetic field. Plasma Physics and Controlled Fusion 54, 105021 (2012).

22. Takabe, H. et al. High-mach number collisionless shock and photo-ionized non-LTE plasma for laboratory astrophysics with intense lasers. Plasma Physics and Controlled Fusion 50, 124057 (2008).

23. Tidman, D. A. \& Krall, N. A. Shock waves in collisionless plasmas (Wiley-Interscience, New York, 1971),

24. Gurevich, A. V. et al. Self-similar motion of rarefied plasma. Soviet Physics JETP 22, 449-454 (1966). 
25. Trubnikov, B. A. Particle Interactions in a Fully Ionized Plasma. Reviews of Plasma Physics 1, 105 (1965).

26. Weibel, E. S. Spontaneously growing transverse waves in a plasma due to an anisotropic velocity distribution. Phys. Rev. Lett. 2, 83-84 (1959).

27. Fried, B. D. Mechanism for Instability of Transverse Plasma Waves. Physics of Fluids 2 , 337-337 (1959).

28. Medvedev, M. V., Fiore, M., Fonseca, R. A., Silva, L. O. \& Mori, W. B. Long-time evolution of magnetic fields in relativistic gamma-ray burst shocks. The Astrophysical Journal 618, L75-L78 (2004).

29. Ruyer, C. \& Fiuza, F. Disruption of current filaments and isotropization of the magnetic field in counterstreaming plasmas. Phys. Rev. Lett. 120, 245002 (2018).

30. Hillas, A. M. The Origin of Ultra-High-Energy Cosmic Rays. Annual Review of Astronomy and Astrophysics 22, 425-444 (1984).

31. In Froula, D. H., Glenzer, S. H., Luhmann, N. C. \& Sheffield, J. (eds.) Plasma Scattering of Electromagnetic Radiation (Second Edition) (Academic Press, Boston, 2011), second edition

32. Mariscal, D. et al. Calibration of proton dispersion for the nif electron positron proton spectrometer (nepps) for short-pulse laser experiments on the nif arc. Review of Scientific Instruments 89, $10 \mathrm{I} 145$ (2018). 
33. Bonnet, T. et al. Response functions of imaging plates to photons, electrons and 4he particles. Review of Scientific Instruments 84, 103510 (2013).

34. Halverson, W. Bremsstrahlung photon emission rate from maxwellian plasmas. Plasma Physics 14, 601-604 (1972).

35. Marinak, M. M. et al. Three-dimensional hydra simulations of national ignition facility targets. Physics of Plasmas 8, 2275-2280 (2001).

36. Fonseca, R. A. et al. OSIRIS: A Three-Dimensional, Fully Relativistic Particle in Cell Code for Modeling Plasma Based Accelerators, 342-351 (Springer Berlin Heidelberg, Berlin, Heidelberg, 2002).

37. Fonseca, R. A. et al. One-to-one direct modeling of experiments and astrophysical scenarios: pushing the envelope on kinetic plasma simulations. Plasma Physics and Controlled Fusion 50, 124034 (2008). 


\begin{tabular}{ccc}
\hline Parameter & NIF experiments & $\begin{array}{c}\text { Typical young SNR } \\
(\text { e.g. SN 1006) }\end{array}$ \\
\hline Shock velocity $(\mathrm{km} / \mathrm{s})$ & $1000-2000$ & $3000-5000$ \\
Ambient magnetic field $(\mathrm{G})$ & $2 \times 10^{4}$ & $3 \times 10^{-6}$ \\
Ambient plasma density $\left(\mathrm{cm}^{-3}\right)$ & $5 \times 10^{19}$ & 0.2 \\
Ambient plasma temperature $(\mathrm{eV})$ & 500 & 1 \\
System size $(\mathrm{cm})$ & 2.5 & $3 \times 10^{19}$ \\
Collisionality $\left(L_{\text {system }} / L_{\text {m.f.p. }}\right)$ & 0.03 & 0.01 \\
Sonic Mach number $\left(v_{\text {sh }} / c_{S}\right)$ & 12 & 400 \\
Alfvén Mach number $\left(v_{\text {sh }} / v_{A}\right)$ & 400 & 400 \\
\hline
\end{tabular}

Table 1: Comparison between plasma parameters in NIF experiments and young SNR shocks. 


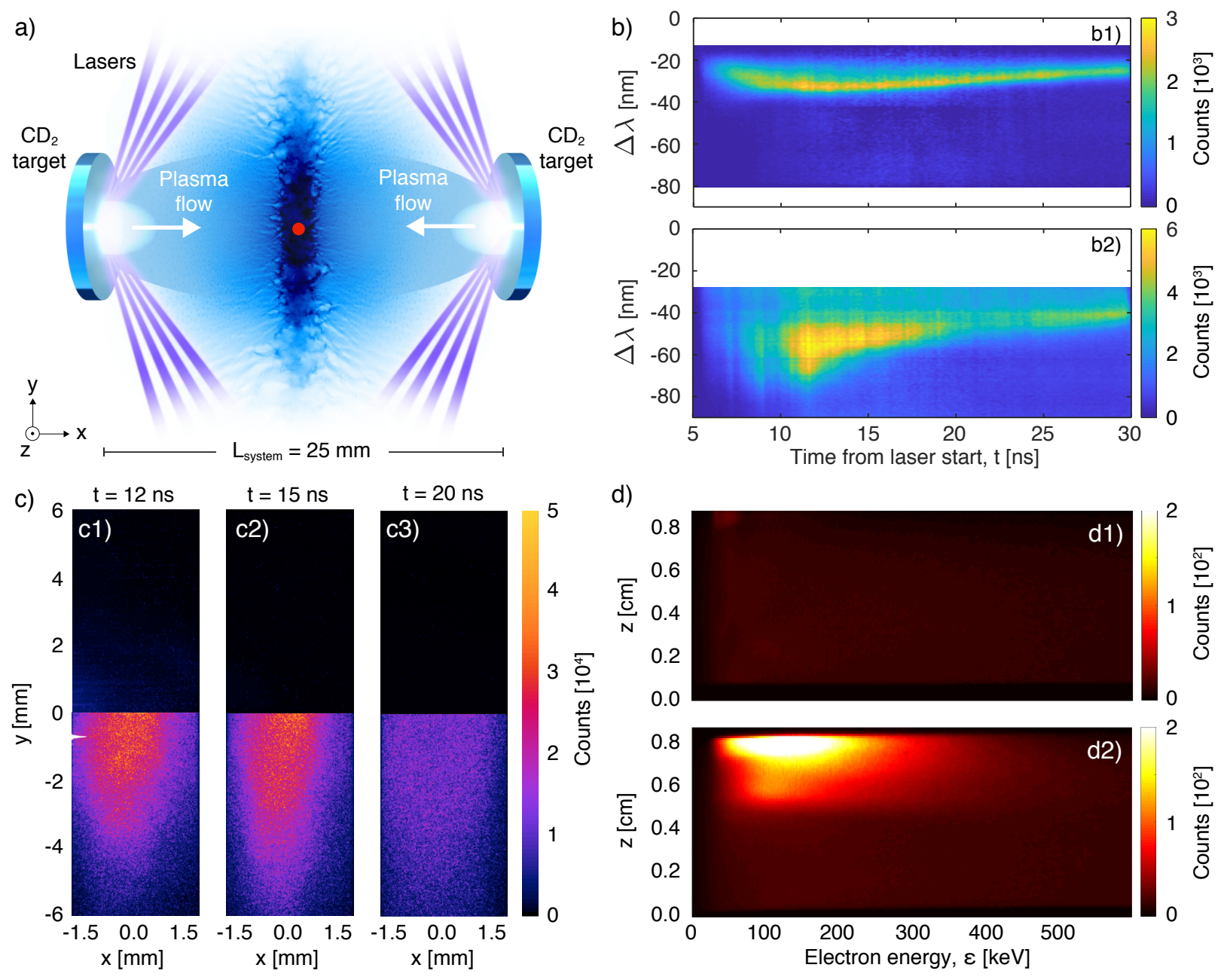

Figure 1: Laser-driven collisionless shock experiments. a) Sketch of the experimental setup with shock density structure (in blue) obtained from numerical simulation. b) Thomson scattering data provides measurement of electron density and temperature at the central region (marked by red dot in a) for a b1) single flow and b2) two colliding flows. c) Comparison of the X-ray selfemission from the plasma between a single flow (top) and colliding flows (bottom) indicates strong compression and heating of the shocked plasma. d) Spectrometer measurements of fast electrons ( $>30 \mathrm{keV}$ ) produced in $\mathrm{d} 1$ ) a single flow and d2) two colliding flows demonstrate acceleration of electrons to relativistic energies. 

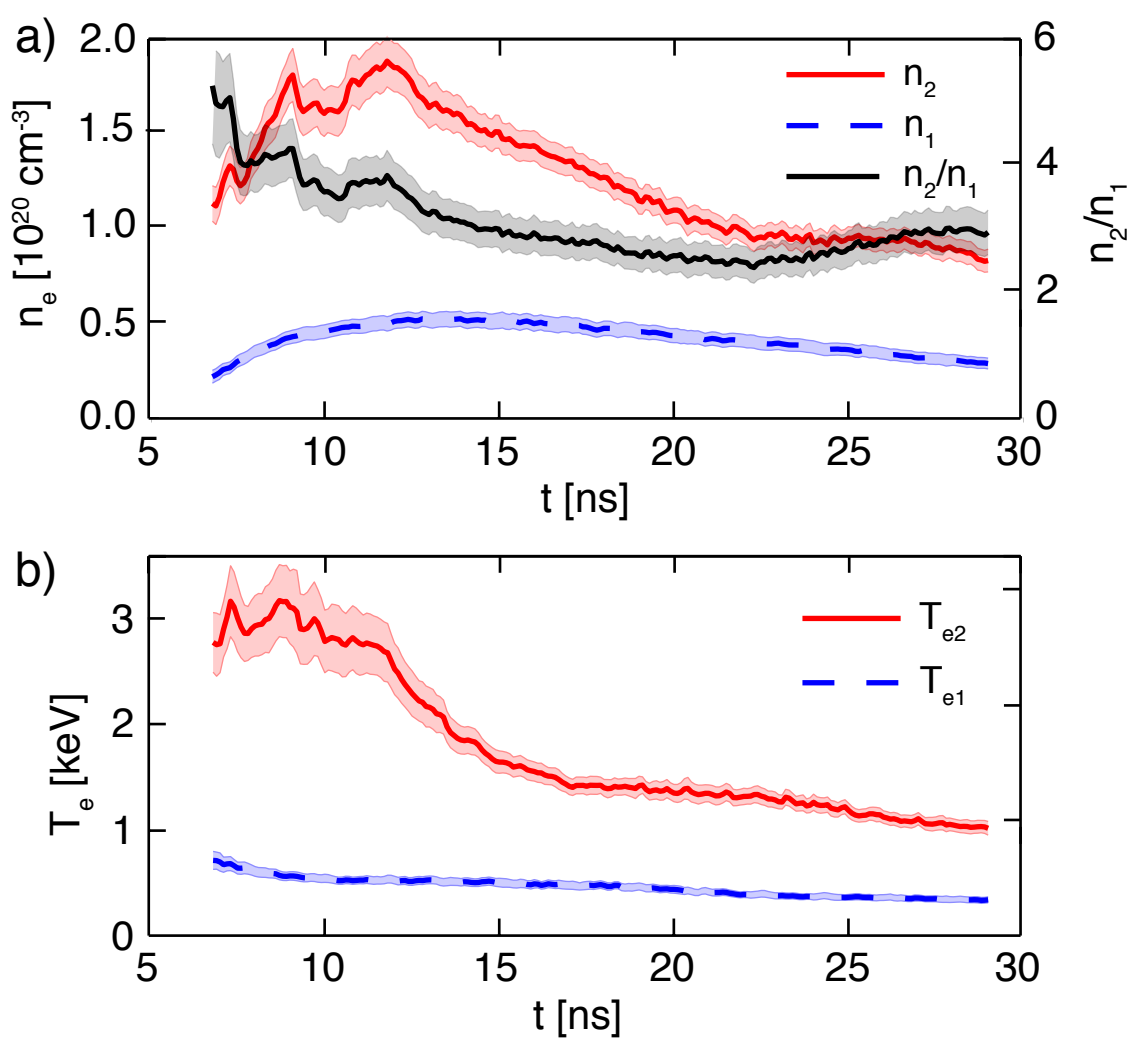

Figure 2: Thomson scattering measurements indicating shock formation. a) Evolution of the electron density at the central region of the experiment for single flow $\left(n_{1}\right.$, dashed blue) and two colliding flows $\left(n_{2}\right.$, solid red). The compression ratio (black) between downstream $\left(n_{2}\right)$ and upstream $\left(n_{1}\right)$ densities reaches $n_{2} / n_{1} \gtrsim 4$ at early times ( $\left.<10 \mathrm{~ns}\right)$ indicating shock formation. b) Evolution of the electron temperature for single flow $\left(T_{e 1}\right.$, dashed blue) and colliding flows $\left(T_{e 2}\right.$, solid red) shows significant heating of the shocked (downstream) electrons to $3 \mathrm{keV}$. As the shocked plasma expands in the transverse direction (see Figure 1a), the compression ratio and plasma temperature slowly decrease in time. The error in the measurements is indicated by the shaded regions and corresponds to the standard deviation of the fit of the Thomson scattering spectrum to the plasma parameters. 
a)
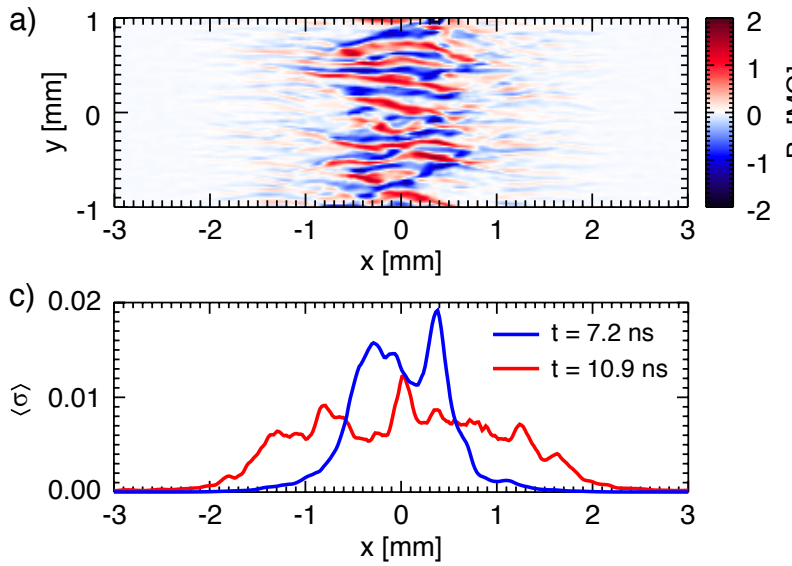
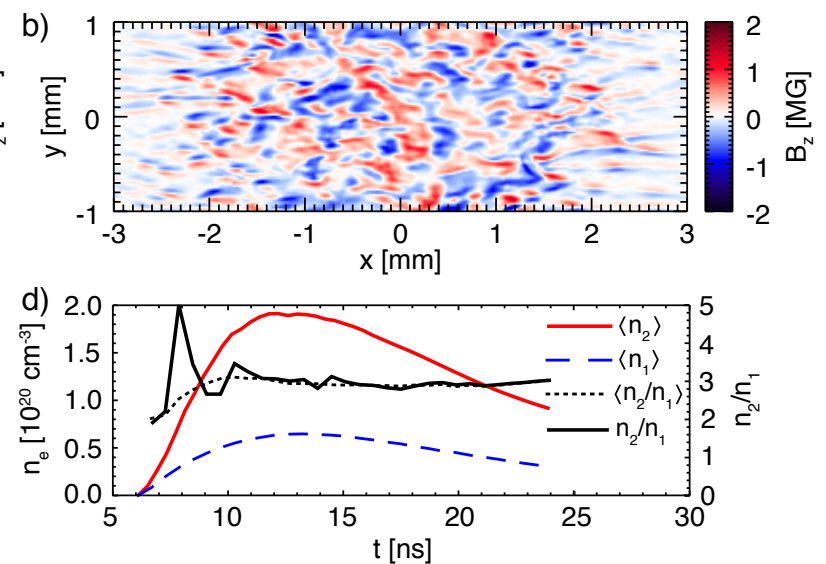

Figure 3: 2D particle-in-cell simulation of the shock structure. Magnetic field maps are shown at a) $7.2 \mathrm{~ns}$ and b) $10.9 \mathrm{~ns}$, indicating the generation of turbulent MG fields associated with the Weibel instability. c) The spatially averaged ratio of magnetic energy density to flow energy density $(\sigma)$ reaches $>1 \%$ in the shocked (downstream) plasma. d) Evolution of the spatially averaged single flow ( $n_{1}$, dashed blue) and double flow ( $n_{2}$, solid red) density at the center of the interaction region. The shock is formed at 9 ns when the compression ratio (dotted black) between downstream $\left(n_{2}\right)$ and upstream $\left(n_{1}\right)$ densities reaches $n_{2} / n_{1} \sim 3$, as predicted from the 2D shock jump conditions (instead of $n_{2} / n_{1} \sim 4$ in 3D). The compression ratio measured in the simulation for a small region corresponding to the Thomson scattering probing volume (solid black) shows large modulations during the shock formation phase $(\lesssim 9 \mathrm{~ns})$, associated with the development of smallscale turbulence. Note that the simulation uses a periodic box transversely and thus cannot capture the transverse expansion of the plasma after shock formation and subsequent decay in density. 

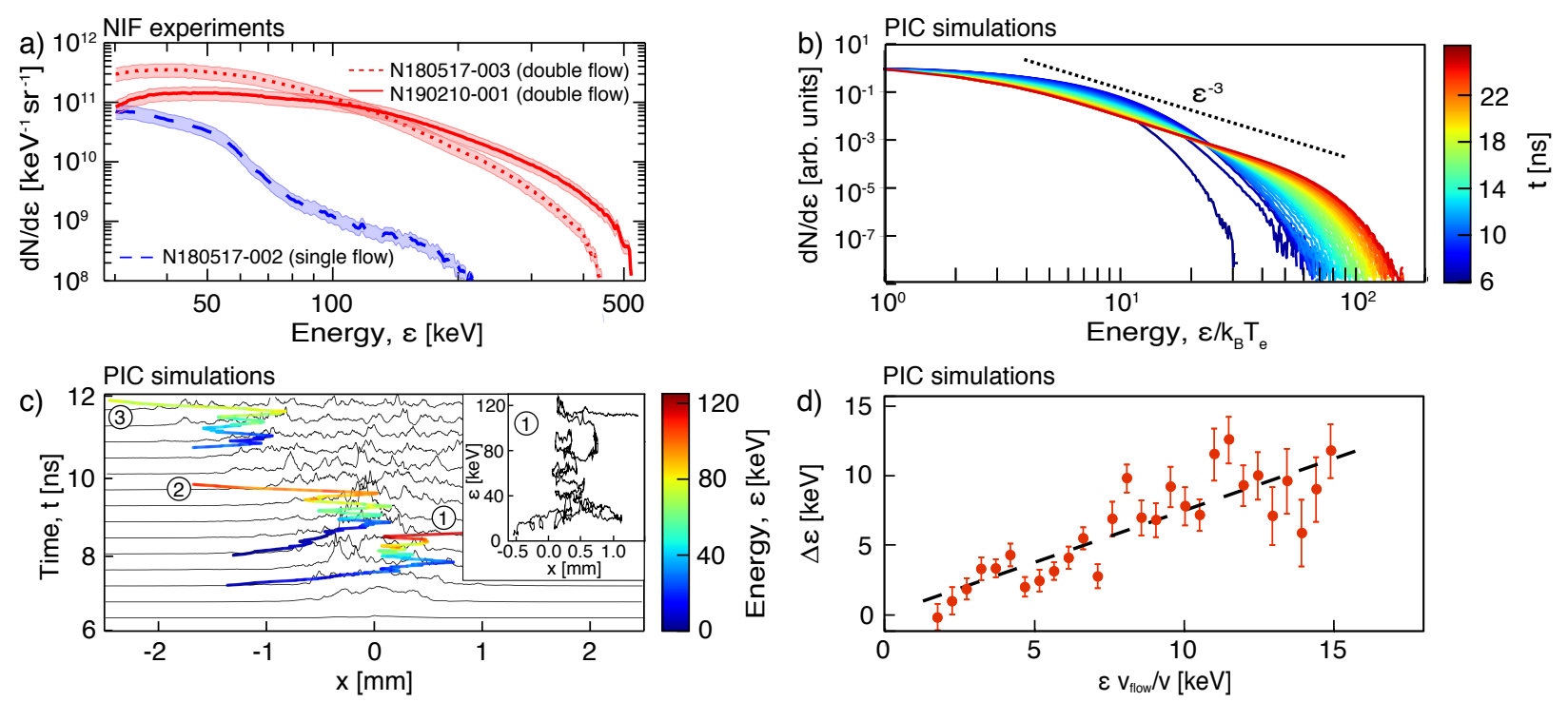

Figure 4: Non-thermal electron acceleration. a) Comparison of the measured time-integrated electron spectrum in double flow (solid and dotted red) and single flow (dashed blue) experiments indicates that when a shock is formed electrons are accelerated up to $500 \mathrm{keV}$. Shaded regions indicate error bars of $\pm 25 \%$ corresponding to a systematic error in the image plate response of the electron spectrometer. b) Evolution of the electron spectrum (colored by time) in the shocked region obtained in simulations confirms that the shock accelerates electrons to $\gtrsim 100 k_{B} T_{e}$, leading to the formation of a non-thermal power-law tail $\epsilon^{-p}$ with a spectral index $p=3$ (dotted). c) Trajectories of three representative non-thermal electrons (colored by energy) overlaid on the magnetic energy profile (black lines) show energization due to stochastic interactions with the turbulent fields within the shock transition. The inset shows that electrons can gain energy via multiple reflections along the flow axis $(x)$ as they are trapped in the shock transition layer. d) The average energy gain experienced by electrons in each reflection (red dots) is consistent with a first-order Fermi process (dashed curve shows linear fit). Error bars indicate the standard error of the mean. 


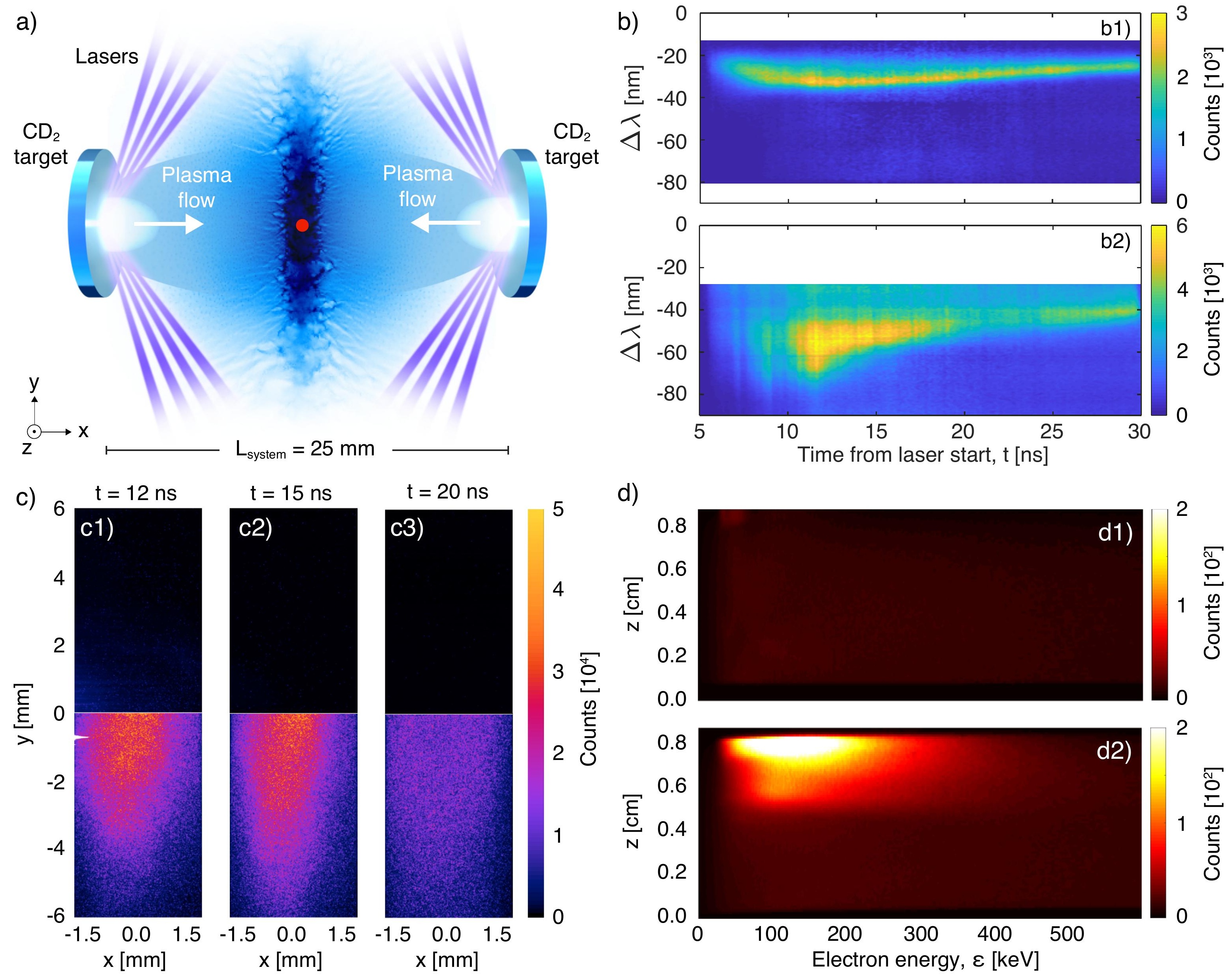




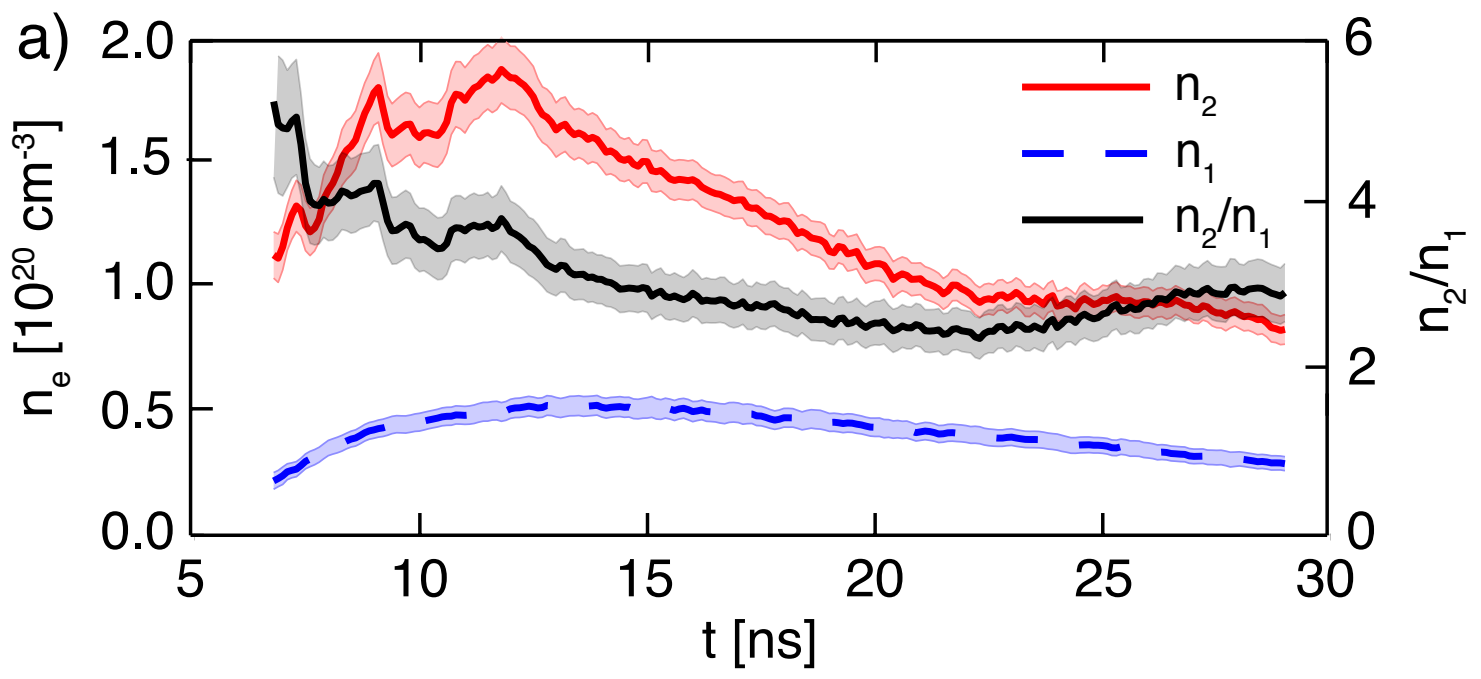

b)

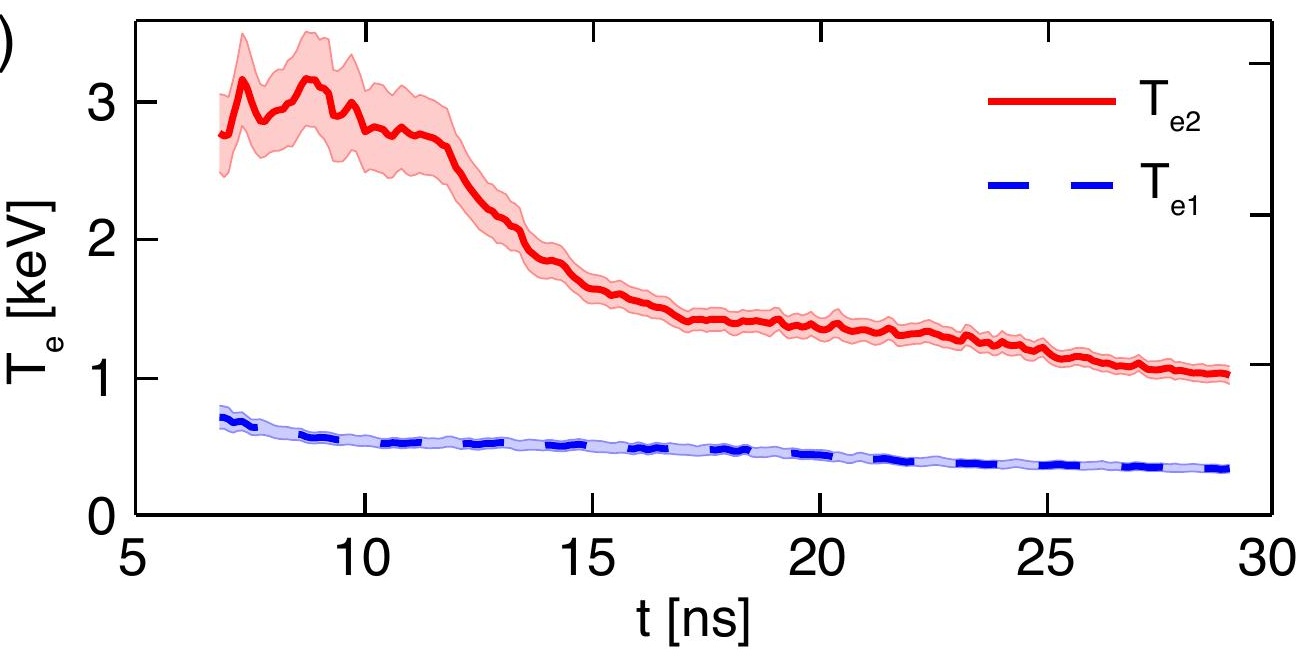



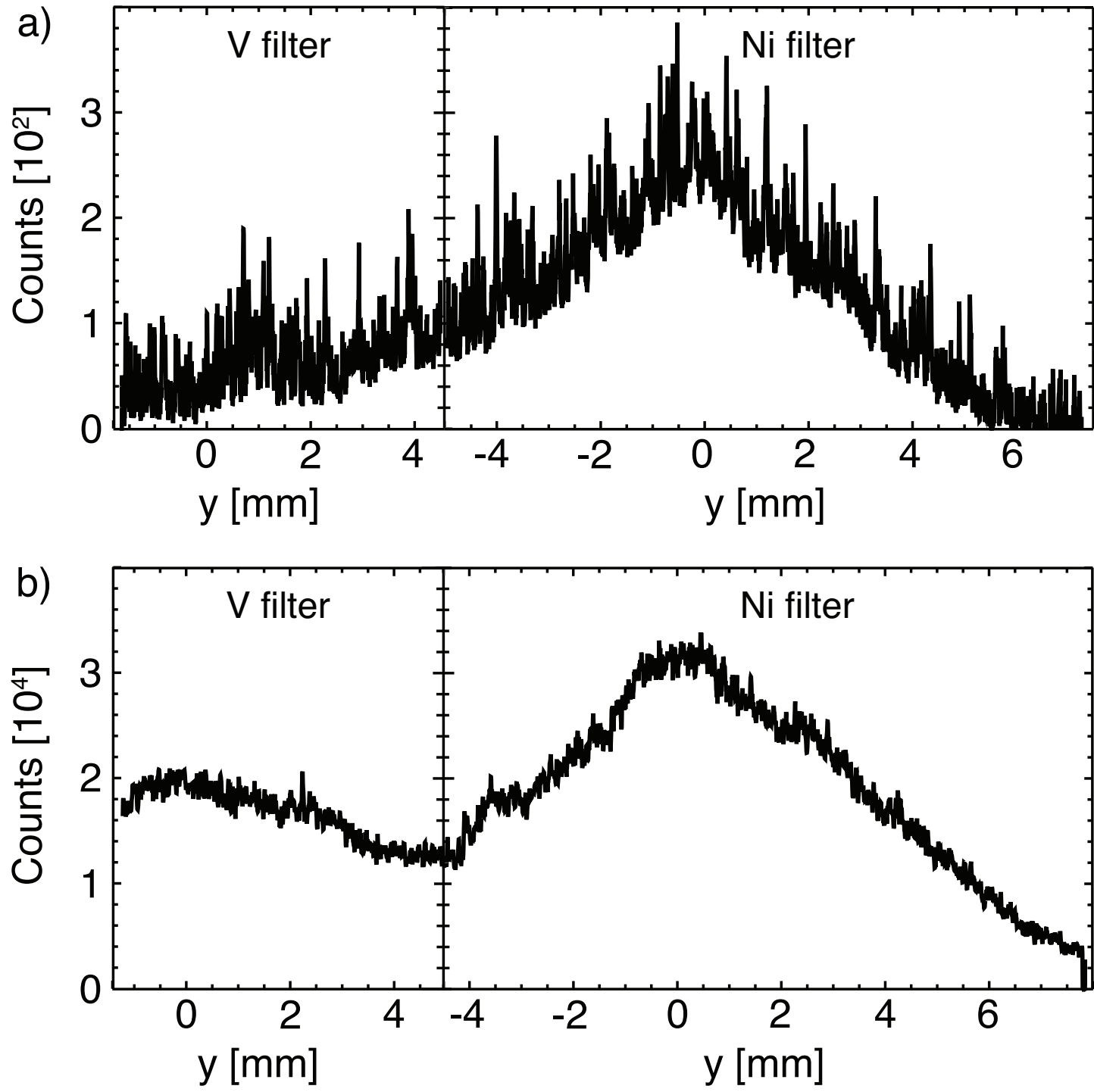